\title{
Atomic-Scale Detection of Organic Molecules Coupled to Single-Walled Carbon Nanotubes
}

\author{
Sung You Hong, ${ }^{\dagger}$ Gerard Tobias, ${ }^{\ddagger}$ Belén Ballesteros, ${ }^{\ddagger}$ Farid El Oualid, ${ }^{\dagger}$ James C. Errey, ${ }^{\dagger}$ \\ Katie J. Doores, ${ }^{\dagger}$ Angus I. Kirkland, ${ }^{\prime}$ Peter D. Nellist, ${ }^{\#}$ Malcolm L. H. Green, ${ }^{*, \ddagger}$ and \\ Benjamin G. Davis*, \\ Department of Chemistry, Chemistry Research Laboratory, University of Oxford, Mansfield Road, Oxford, \\ OX1 3TA, U.K., Inorganic Chemistry Laboratory, University of Oxford, South Parks Road, Oxford, OX1 3QR U.K., \\ and Department of Materials, University of Oxford, Parks Road, Oxford, OX1 3PH, U.K.
}

Received December 19, 2006; E-mail: malcolm.green@chem.ox.ac.uk; ben.davis@chem.ox.ac.uk

Functionalized carbon nanotubes (f-CNTs) bearing organic molecules and biomolecules show strong potential in biological and biomedical applications. ${ }^{1-3}$ Suitable covalent functionalization of single-walled carbon nanotubes (SWNTs) can tailor critical properties such as solubility in solvents including water, which is crucial for biological applications. ${ }^{4,5}$ Bulk measurements (Raman, IR, UV) are commonly used to characterize modified SWNTs; however, these do not provide direct evidence of the attachment of individual molecules.

High-resolution transmission electron microscopy (HRTEM) and scanning TEM (STEM) are the most powerful techniques for direct imaging and analysis of inorganic nanostructures and provide structural information down to the subnanometer range. Moreover, when coupled with energy-dispersive X-ray spectroscopy (EDS) and electron energy-loss spectroscopy (EELS), chemical and electronic data can also be collected. ${ }^{6-8}$ In contrast, use of HRTEM/ STEM for organic compounds is limited by presentation as individual molecules without structural periodicity and typically contain low-scattering elements $(\mathrm{H}, \mathrm{C}, \mathrm{N}, \mathrm{O}) .{ }^{9}$ Moreover, organic molecules may be easily confused with amorphous carbon partially covering the surfaces of SWNTs.

An important step toward the direct imaging of organic compounds would be their redesign by introducing high scattering heavy elements, which are clearly visible in HRTEM/STEM. ${ }^{10}$ Here we present for the first time the synthesis and modification of organic molecules covalently bonded to SWNTs for their detection via HRTEM/STEM in the atomic scale. In the present study, carbohydrates have been the organic molecule of choice. Because of their complex structures, carbohydrates are unrivaled in the density of information that they can convey and act as markers in important recognition processes. ${ }^{11-13}$ They are also representative models for polyfunctional organic molecules.

Two different approaches were investigated. In the sequential route, carboxylates on oxidized SWNTs (SWNT 1) were preactivated by DCC/HOBt prior to direct Staudinger coupling, ${ }^{14}$ with putative iminophosphorane derived from GlcNAc-azide 5. ${ }^{14}$ 2,3,5Triiodobenzoyl (TIBz) was selected as a high scattering-tagged variant of the widely used benzoyl $(\mathrm{Bz})$ protecting group. It was appended to carbohydrate-coupled SWNT 2 under Steglich conditions, ${ }^{15}$ which allowed successful, although partial, esterification. As an alternative, convergent method, tri-TIBz-GlcNAc azide 4, from reaction of $\mathbf{5}$ and TIBz-chloride, was coupled ${ }^{14}$ directly to SWNT 1 and allowed higher levels of glycan incorporation.

TIBz-tags valuably present heavy atoms at well-defined distances free of conformational ambiguity. HRTEM, EDS, and STEM of

\footnotetext{
$\dagger$ Department of Chemistry.

¥ Inorganic Chemistry Laboratory.

\# Department of Materials.
}

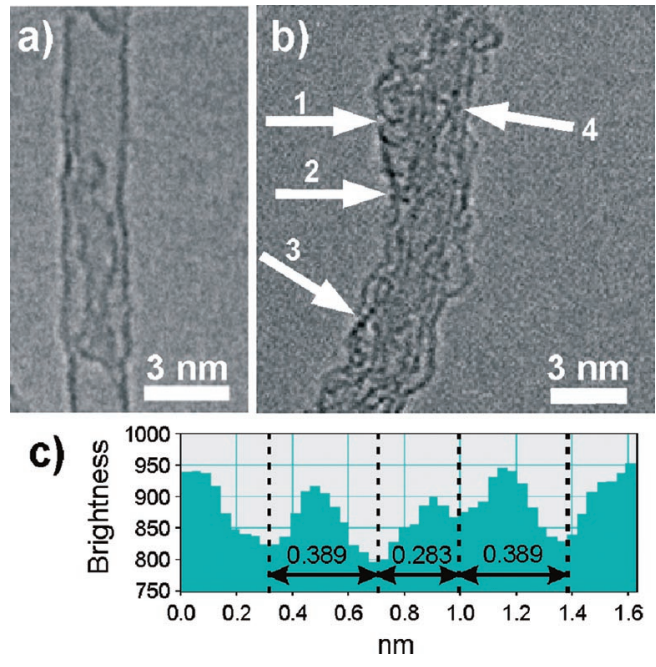

Figure 1. HRTEM images of (a) purified SWNT and (b) SWNT 3 from sequential route showing iodides along the sidewall; (c) line profile integrated along iodine atoms indicated by arrow $3 .{ }^{16}$

iodide-tagged SWNT 3 clearly revealed (Figures 1 and 2) the presence and significant level of iodide through functionalization by TIBz. Direct imaging of individual iodides by Z-contrast STEM was possible because a large contrast is observed between I $(Z=$ 53) and $\mathrm{C}, \mathrm{N}, \mathrm{O}(\mathrm{Z}=6,7,8$, respectively) as a result of the Rutherford scattering used to form $Z$-contrast images which scales approximately with the square of the atomic number. ${ }^{10}$ Areas where SWNT walls are clean by bright-field imaging also show no iodide by dark-field imaging; many bright dots (iodides) are observed on functionalized areas. We believe that this is the first example of unambiguous atomic-scale detection of organic molecules on SWNTs. Advantageously, like its nontagged counterpart, Bz, tagged protecting group TIBz was readily cleaved; Zemplén deesterfication led to clean restoration of hydroxyl groups. As anticipated, negligible iodide was found in the resulting sample, thereby confirming the switchable nature of the tagged (SWNT 3) $\leftrightarrow$ untagged (SWNT 2) interconversions (Scheme 1).

Functionalization of SWNTs was further confirmed by FTIR and NMR spectroscopy (see Supporting Information): carboxylic acid after nitric acid treatment $\left(1720-1740 \mathrm{~cm}^{-1}\right) ; \mathrm{C}=\mathrm{O}$ amide after Staudinger reaction of 5 to give $2\left(1610-1660 \mathrm{~cm}^{-1}\right.$, amide I). When tri-TIBz-GlcNAcN ${ }_{3} \mathbf{4}$ was coupled to SWNT 1 to give SWNT 3, amide I bands appeared with strengthening of $\mathrm{C}=\mathrm{O}$ bands $\left(1720-1740 \mathrm{~cm}^{-1}\right)$ and bands at $1510-1530 \mathrm{~cm}^{-1}$ for TIBz $\mathrm{C}=\mathrm{C}$ stretching and amide II. ${ }^{1} \mathrm{H}$ NMR resonances of iodide-tagged SWNT 3 were broader than for tri-TIBz-GlcNAcN $\mathrm{N}_{3} \mathbf{4}$, consistent with lower tumbling frequency. ${ }^{17,18}$ 

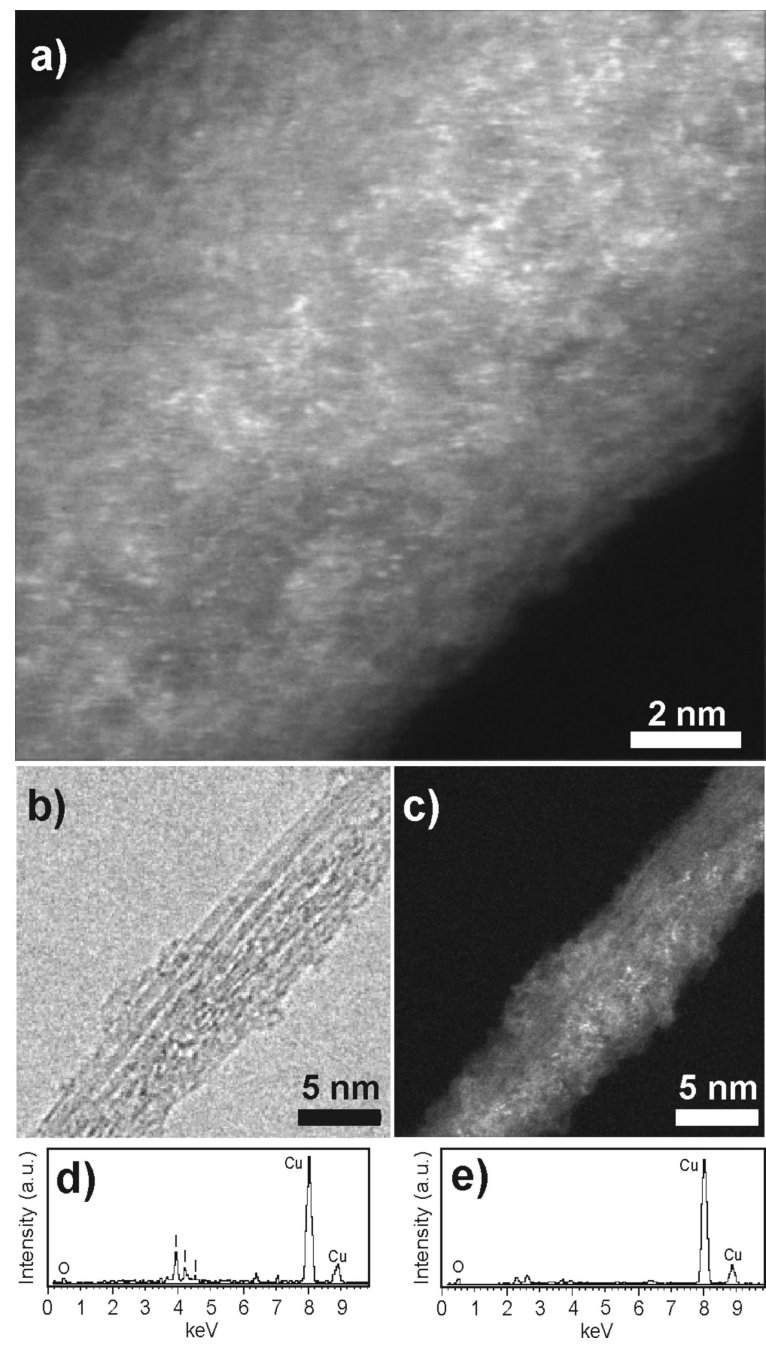

Figure 2. (a) Z-contrast dark field STEM images of SWNT 3; individual iodides (white dots) can be clearly seen. (b) STEM bright field phase contrast image and (c) annular dark field Z-contrast image of same region of SWNT 3 clearly show presence of iodide (bright dots) where the SWNTs are functionalized (more images in Supporting Information). (d, e) EDS spectra of iodide tagged SWNT 3 before (d) and after (e) Zemplén deesterification.

Scheme 1. Sequential and Convergent Syntheses of I-Tagged SWNTa

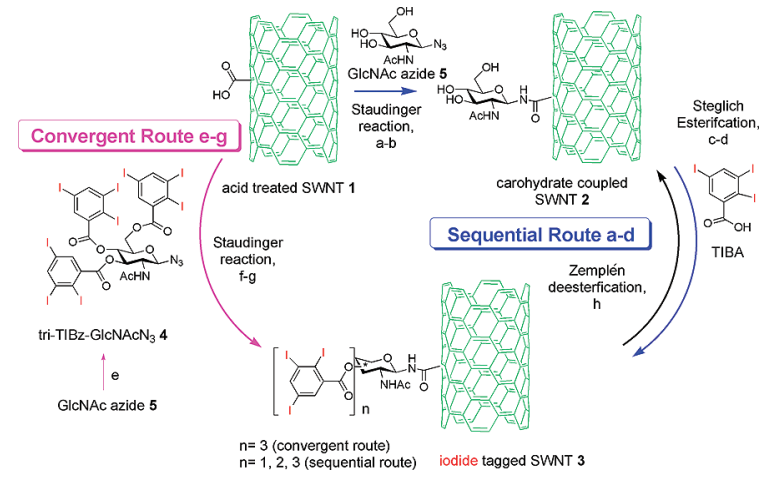

${ }^{a}$ Conditions: (a) SWNT 1, DCC/HOBt, DMF; (b) GlcNAcN ${ }_{3}$ 5, $\mathrm{PBu}_{3}$, DMF; (c) TIBA, EDC/DMAP, DMSO; (d) SWNT 2, DMSO; (e) 2,3,5triiodobenzoyl chloride, pyridine, $74 \%$; (f) $\mathrm{SWNT} 1, \mathrm{SOCl}_{2}, \Delta$; (g) triTIBz-GlcNAcN ${ }_{3}$ 4, $\mathrm{PBu}_{3}$, DMF; (h) NaOMe, $\mathrm{MeOH}$, DMSO.

Finally, as an additional key test of not only the presence of unprotected sugar GlcNAc but also of such precisely functionalized SWNTs to interact with carbohydrate-active proteins we tested glyco-SWNT 2 as a non-natural macromolecular substrate for a glycosyltransferase. We were pleased to find $\mathbf{2}$ was soluble and active in assays with $\beta 1$,4-galactosyltransferase, at levels consistent with its GlcNAc loading. ${ }^{19}$ This represents the first example of an enzymatic glycosylation on the surface of a SWNT; moreover transfer of $\left[6-{ }^{3} \mathrm{H}\right]-\mathrm{Gal}$ to GlcNAc allowed controlled, site-selective radiolabeling of SWNT 2

In summary, we have demonstrated that rational design of organic molecules containing heavy element tags allows direct detection by HRTEM/STEM in the subnanometer scale. Using this method we have presented direct evidence of functionalization of SWNTs with carbohydrates (glyco-SWNTs). ${ }^{5,20}$ Given difficulties associated with verification of such organic functionalization on SWNTs we believe that such heavy element tagging-mediated direct detection could play a valuable role. Furthermore, protecting group manipulation in these SWNTs may be achieved and directly detected using HRTEM/STEM thereby allowing precise manipulation of hydroxyl groups on these glyco-SWNTs, which are key recognition determinants. The successful interaction of these precisely constructed glyco-SWNTs with carbohydrate-active proteins demonstrates, in principle, utility as probes of biological processes. It is also hoped that this visual detection method might stimulate development of other high scattering elements tagging methods.

Acknowledgment. The authors thank Drs. C. G. Salzmann, Y. Huh, and M. Yang for assistance; Thomas Swan \& Co. Ltd. for SWNTs and funding; Samsung Corporation (fellowship S.Y.H.); FP6 Marie Curie IE Fellowship MEIF-CT-2006-024542 (G.T.); and EPSRC (Basic Technology GR/S83968/01).

Supporting Information Available: Experimental procedures and characterization of SWNTs. This material is available free of charge via the Internet at http://pubs.acs.org.

\section{References}

(1) Wu, W.; Wieckowski, S.; Pastorin, G.; Benincasa, M.; Klumpp, C.; Briand, J. P.; Gennaro, R.; Prato, M.; Bianco, A. Angew. Chem., Int. Ed. 2005 44, 6358-6362.

(2) Williams, K. A.; Veenhuizen, P. T. M.; de la Torre, B. G.; Eritja, R.; Dekker, C. Nature 2002, 420, 761-761.

(3) Yim, T. J.; Liu, J. W.; Lu, Y.; Kane, R. S.; Dordick, J. S. J. Am. Chem. Soc. 2005, 127, 12200-12201.

(4) Pantarotto, D.; Partidos, C. D.; Graff, R.; Hoebeke, J.; Briand, J. P.; Prato, M.; Bianco, A. J. Am. Chem. Soc. 2003, 125, 6160-6164.

(5) Hirsch, A. Angew. Chem., Int. Ed. 2002, 41, 1853-1859.

(6) Iijima, S. Nature 1991, 354, 56-58

(7) Margulis, L.; Salitra, G.; Tenne, R.; Talianker, M. Nature 1993, 365, $113-$ 114

(8) Fernandez-Garcia, M.; Martinez-Arias, A.; Hanson, J. C.; Rodriguez, J. A. Chem. Rev. 2004, 104, 4063-4104

(9) Coleman, K. S.; Bailey, S. R.; Fogden, S.; Green, M. L. H. J. Am. Chem. Soc. 2003, 125, 8722-8723.

(10) (a) Meyer, R. R.; Sloan, J.; Dunin-Borkowski, R. E.; Kirkland, A. I.; Novotny, M. C.; Bailey, S. R.; Hutchison, J. L.; Green, M. L. H. Science 2000, 289, 1324-1326. (b) Crewe, A. V.; Wall, J.; Langmore, J. Science 1970, 168 1338-1340. (c) Nellist, P. D.; Pennycook, S. J. Science 1996 $274,413-415$

(11) Davis, B. G. Chem. Rev. 2002, 102, 579-601.

(12) Dwek, R. A. Chem. Rev. 1996, 96, 683-720.

(13) Varki, A. Glycobiology 1993, 3, 97-130.

(14) Doores, K. J.; Mimura, Y.; Dwek, R. A.; Rudd, P. M.; Elliott, T.; Davis, B. G. Chem. Commun. 2006, 1401-1403.

(15) Neises, B.; Steglich, W. Angew. Chem., Int. Ed. 1978, 17, 522-524.

(16) Hong, S. Y.; Popovitz-Biro, R.; Prior, Y.; Tenne, R. J. Am. Chem. Soc 2003, 125, 10470-10474

(17) Gu, L. R.; Lin, Y.; Qu, L. W.; Sun, Y. P. Biomacromolecules 2006, 7, $400-402$.

(18) Sun, Y. P.; Fu, K. F.; Lin, Y.; Huang, W. J. Acc. Chem. Res. 2002, 35, 1096-1104.

(19) Loadings and transformation efficiencies of $0.014-0.025( \pm 0.007) \mathrm{mmol} / \mathrm{g}$ for deprotection $(>95 \%)$ and protection $(>80 \%)$ were estimated using GalT endpoint analysis and EDS abundance data using the tagging approach.

(20) We advocate combined characterization: heavy-atom tagged protecting groups enable use of EDS, HRTEM, and STEM and are valuably supplemented by IR or NMR spectroscopy in cases where access to TEM methods may be limited.

JA069080I 